\title{
The Central Radio Gap and the Equatorial Emission Region in SS433
}

\author{
Z. Paragi and I. Fejes \\ FÖMI Satellite Geodetic Observatory, H-1373 Budapest, P.O. Box 546, \\ Hungary \\ R.C. Vermeulen, R.T. Schilizzi, R.E. Spencer and A.M. Stirling \\ NFRA, The Netherlands, JIVE and Leiden Obs., The Netherlands, \\ NRAL, United Kingdom, Univ. Central Lancashire, United Kingdom
}

\begin{abstract}
.
The radio-jet X-ray binary SS433 was observed at five epochs in 1998 by the very long baseline interferometry (VLBI) technique at five frequencies ranging from 1.6 to $22 \mathrm{GHz}$. The innermost region of the source on milliarcsecond scales $(1 \mathrm{mas}=5 \mathrm{AU})$ is resolved into an eastern and a western core-jet component, well separated by the Central Radio Gap (25-30 AU projected size), where the binary stellar system is located. We suggest that the radio gap is caused by local synchrotron self-absorption and external free-free absorption in an ionized medium, which has a disk-like geometry. On $100 \mathrm{AU}$ scales we observe the Equatorial Emission Region, oriented roughly perpendicularly to the jets, with variable morphology at different epochs. Both of these phenomena could be interpreted with a mass outflow from the system, concentrated in the orbital plane of the binary.
\end{abstract}

\section{The Central Radio Gap}

The radio core of SS433 is not centre brightened, but has a double core-wing morphology (Vermeulen et al. 1993). Multi-frequency observations with the VLBA in 1995 showed that there is a radio gap between the approaching and receding jet sides at 5 and $15 \mathrm{GHz}$ (Paragi et al. 1999), hereafter Central Radio Gap. Such a region was suggested to be present in SS433 by Stirling, Spencer, \& Watson (1997) due to free-free absorption in the ionized stellar wind, but also expected according to conical jet models if the synchrotron self-absorption optical depth is high enough (cf. Blandford \& Königl 1979 and Hjellming \& Johnston 1988). Kinematic modelling revealed that the central engine (i.e. the binary stellar system) is located within the Central Radio Gap. The size of the gap depends on both time and frequency. One expects that the Central Radio Gap decreases in extent with increasing frequency due a decrease in opacity. This is observed between 1.6 and $5 \mathrm{GHz}$, where synchrotron self-absorption dominates. But at higher frequencies the core-jet separation between the approaching and receding jet sides does not seem to decrease below 5-6 mas 
(25-30 AU projected linear size at the distance of the source). This, and the observed brightness asymmetry with increasing frequency indicate the presence of a disk-like free-free absorbing medium, probably related to an outflow from the system. This outflow cannot be spherically symmetric, as this would invoke an unexpectedly high mass outflow rate of $10^{-5} \mathrm{M}_{\odot} /$ year.

\section{The Equatorial Emission Region}

Two radio components were discovered at an anomalous position angle in SS433, after analyzing the $1.6 \mathrm{GHz}$ data taken in 1995 . These were located roughly perpendicular to the radio beams, and spanning several hundred AU in a disposition that was quasi-symmetric to the Central Radio Gap (Paragi et al. 1999). We confirm the existence of this radio emitting region in the 1998 observations. As the "anomalous" radio components cannot be related directly to the precessing radio beams, which were ejected near the poles of the compact object, we refer to the region where these appear as the Equatorial Emission Region. The observed high brightness temperatures of $10^{7}-10^{8} \mathrm{~K}$ exclude a thermal origin for the emission. The region changes with time, but probably on a much longer timescale than the radio beams. The Equatorial Emission Region might be fed by a disk-like outflow from the system, or by the strong stellar wind, concentrated into the orbital plane of the binary.

\section{Conclusions}

The Central Radio Gap is caused by a combined effect of synchrotron selfabsorption (according to conical jet models) and free-free absorption. The geometry of the external ionized medium is probably disk-like; it might be related to an outflow from the system. This outflow may feed the Equatorial Emission Region, but the emission mechanism is unclear at present.

Acknowledgments. Z.P. acknowledges financial support received from the International Astronomical Union (IAU) to attend the IAU 205 Symposium. This research was financially supported by the Hungarian Space Office (MÜI), the Netherlands Organization for Scientific Research (NWO) and the Hungarian Scientific Research Fund (OTKA, grant no. N31721 \& T031723).

\section{References}

Blandford, R.D. \& Königl, A. 1979, ApJ, 232, 34

Hjellming, R.M. \& Johnston, K.J. 1988, ApJ, 328, 600

Paragi, Z., Vermeulen, R.C., Fejes, I., Schilizzi, R.T., Spencer, R.E., \& Stirling, A.M. 1999, A\&A, 348, 910

Stirling, A.M., Spencer, R.E., \& Watson, S.K. 1997

Vermeulen, R.C., Schilizzi, R.T., Spencer, R.E., Romney, J.D., \& Fejes I. 1993, A\&A, 270, 177 